\title{
Reviewing the FRP Strengthening Systems
}

\author{
Seyyed Mohammad Banijamali, ${ }^{1,}$, Mohammad Reza Esfahani ${ }^{1}$, Shoeib Nosratollahi ${ }^{2}$ Mohammad \\ Reza Sohrabi $^{2}$, Seyyed Roohollah Mousavi
}

${ }^{1}$ Dept. of Civil Engineering, Ferdowsi University of Mashhad (FUM), Mashhad, Iran

${ }^{2}$ Dept. of Civil Engineering, University of Sistan and Baluchestan (USB), Zahedan, Iran

\section{Email addresses:}

Banijamali.sm@gmail.com (S. M. Banijamali), Esfahani@um.ac.ir (M. R. Esfahani), Shoeib.nosratollahi@yahoo.com (S. Nosratollahi), Sohrabi@hamoon.usb.ac.ir (R. Sohrabi), S.R.Mousavi@eng.usb.ac.ir (S. R. Mousavi)

\section{To cite this article:}

Seyyed Mohammad Banijamali, Mohammad Reza Esfahani, Shoeib Nosratollahi, Mohammad Reza Sohrabi, Seyyed Roohollah Mousavi. Reviewing the FRP Strengthening Systems. American Journal of Civil Engineering. Special Issue: Research and Practices of Civil Engineering in Developing Countries. Vol. 3, No. 2-2, 2015, pp. 38-43. doi: 10.11648/j.ajce.s.2015030202.18

\begin{abstract}
Several methods have been invented for flexural strengthening of RC beams using the FRP materials in recent years. These techniques are categorized into two main groups: Externally Bonded Reinforcement (EBR) techniques and Near Surface Mounted (NSM) techniques. The EBR family contains EBR (with conventional surface preparation), EBROG, EBRIG, MF-EBR, HOLING methods. The NSM family contains NSM-FRP Rods, NSM-FRP Sheets, NSM-MMFRP Rods methods. The EBR family techniques Despite the ease of implementation have weaknesses such as vulnerability against sever environmental conditions. Although the NSM techniques, have longer installation time than the EBR family techniques but in these methods the strengthening materials are greatly protected against the environmental effects. In this paper, the various techniques for flexural strengthening of RC members from both the EBR and NSM families have been fully described and advantages and disadvantages of each technique have been discussed.
\end{abstract}

Keywords: Externally Bonded Reinforcement, Near Surface Mounted, Fiber Reinforced Polymers

\section{Introduction}

Replacing the existing structures with the new structures is often not economically cost effective, therefore finding an appropriate solution for repairing and strengthening structures has a great importance. In comparison to building a new structure, strengthening an existing structure is often more complicated, since the structural conditions are already set. In addition, it is not always easy to reach the areas that need to be strengthened. The improper choice of an inappropriate repair or strengthening method can even worsen structure`s function. Traditional methods have been used as strengthening techniques for concrete structures, such as: different kinds of reinforced overlays, shotcrete or post-tensioned cables placed on the outside of the structure which normally need much space $[1,2]$.

The use of Fiber Reinforced Polymers (FRP) in the last few years in various engineering applications, forums and configurations offers an alternative design approach for the construction of new concrete structures [3] and rehabilitation of existing ones [4]. The key properties that make FRP materials suitable for structural strengthening are their non-corrodible nature and high strength-to-weight ratio. Hence extensive researches on the development and use of these materials have been or are being conducted. The result of these researches is the emergence of different techniques, such as: EBR1, EBROG2, EBRIG3, MF-EBR4, NSM5-FRP rods, NSM-FRP strips, NSM-MMFRP 6rods and HOLING methods for strengthening concrete structures. In this paper each of these techniques will be introduced and their advantages and disadvantages will be discussed.

\section{The EBR method}

\subsection{General features}

Externally Bonded Reinforcement (EBR) technique is the

\footnotetext{
1 Externally Bonded Reinforcement

2 Externally Bonded Reinforcement On Grooves

3 Externally Bonded Reinforcement In Grooves

4 Mechanically Fastened and Externally Bonded Reinforcement

5 Near Surface Mounted

6 Manually Made Fiber Reinforced Polymers
} 
most common method to strengthen reinforced concrete structures. In this method, after surface preparation, FRP sheet is adhesively bonded to the tension face of the concrete member. The purpose of surface preparation is to remove contamination and weak surface layers and polish the concrete surface to promote its adherence capacity. However, the main deficiencies in the performance of EBR technique are high possibility of brittle failure mode that is mostly due to premature debonding of FRP sheet from the concrete substrate and vulnerability of FRP materials against the environmental conditions [5, 6]. Provision of adequate end anchorages at the ends of the plates and at critical sections along the span such as: U, L and $\mathrm{X}$ shaped wrappings and various surface preparation processes such as: surface roughening by sandblast, water jet and air jet, can slightly postpone the debonding phenomenon [7].

"Fig. 1" shows a strengthened cross-section with EBR method.

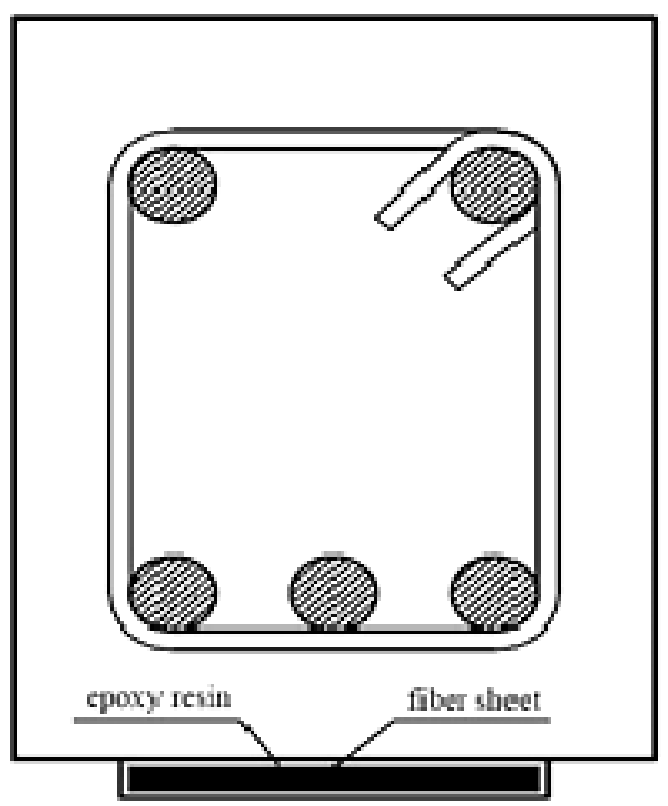

Figure 1. A strengthened cross section with EBR method

\subsection{Advantages}

a) Quick and easy installation

b) Low performing costs

c) Immediate use of strengthened structures

d) No need to specific labor skills

\subsection{Disadvantages}

a) Brittle failure mode due to premature debonding of FRP sheet from the concrete substrate

b) Vulnerability of FRP materials against the environmental conditions such as: freeze/thaw cycles, abrasion, mechanical impacts, acidic and alkaline environments, fire, vandalism and UV radiations.

c) Changes caused in the appearance of the structure

\section{The EBROG method}

\subsection{General features}

Mostofinejad and Mahmoudabadi [8] invented the grooving method (GM) to postpone or even eliminate the debonding of FRP sheets. This technique was later renamed to Externally Bonded Reinforcement On Grooves (EBROG) method. In this method first grooves are cut onto the tension side of the concrete member. These grooves are then cleaned by the air jet and filled with the epoxy resin. FRP sheets are later installed on the concrete surface saturated with the epoxy resin and the resin in excess is removed. Based on their experimental studies, longitudinal grooves are effective than transverse and diagonal grooves, and can be used as an alternative to conventional surface preparation methods [8].

"Fig.2" shows a strengthened cross section with EBROG method.

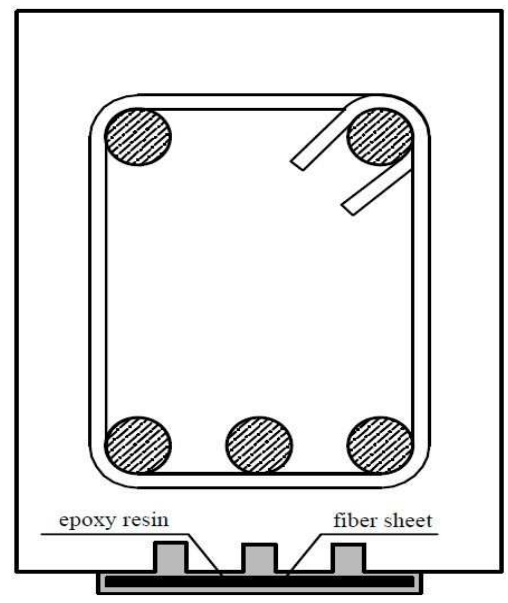

Figure 2. A strengthened cross section with EBROG method

\subsection{Advantages}

a) Increase in the flexural capacity up to $80 \%$ more than reference specimen

b) Achieving the higher ultimate strain

c) Postponing or even eliminating the debonding phenomenon

d) Immediate use of strengthened structure

\subsection{Disadvantages}

a) Time-consuming installation process

b) Costly performing

c) Vulnerability of FRP materials against the environmental conditions

d) Environmental pollution caused by the grooving process

e) Change caused in the appearance of the structure

\section{The EBRIG Method}

\subsection{General Features}

Mostofinejad and Shameli [9] invented an improved grooving method by penetrating the FRP sheets used in the 
EBROG method into the grooves. This technique was named Externally Bonded Reinforcement In Grooves (EBRIG) method. Due to the fact that EBRIG technique provides larger contact area between FRP and the underlying concrete layer compared to EBROG method, this technique significantly promote the structural performance, modify crack initiation and propagation, and increase the failure limits. Based on their result the EBRIG method is effective than EBROG and EBR method when multilayer FRP sheet are used [9].

"Fig.3" shows a strengthened cross section with EBRIG method.

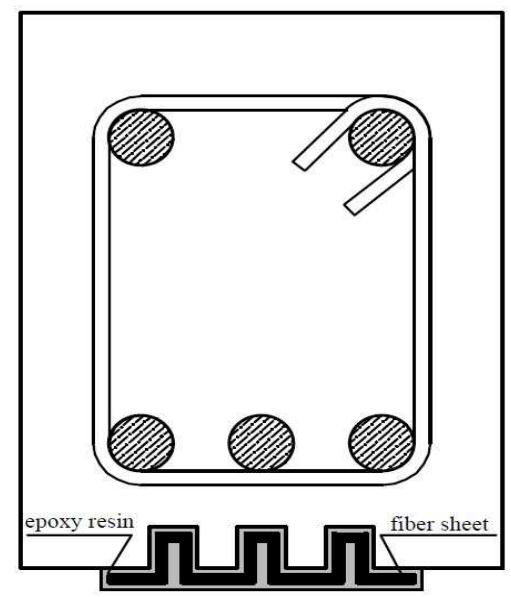

Figure 3. A strengthened cross section with EBRIG method

\subsection{Advantages}

a) Achieving the ultimate loads higher than the previous methods (up to $142 \%$ increase in ultimate load compared to the reference specimen)

b) Achieving the higher ultimate strain

c) Completely eliminating the debonding phenomenon in specimens strengthened with only one layer of FRP sheets.

d) Immediate use of strengthened structures

\subsection{Disadvantages}

a) Time-consuming installation process

b) Costly performing process

c) Vulnerability of FRP materials against the environmental conditions

d) Environmental pollution caused by the grooving process

e) Changes caused in the appearance of the structure

\section{The MF-EBR method}

\subsection{General Features}

Sena-Cruz et al [10] proposed the Mechanically Fastened and Externally Bonded Reinforcement (MF-EBR) method. This technique is based on the MF-FRP method and combines the mechanical Fastening of MF-FRP method with the external adhesion in EBR method. Moreover, all of the anchors are also pre-tensioned $[10,11]$. The strengthening process in- volves the following main steps:

a) Holes of specific diameter depth are made in the soffit of the beam. The holes are cleaned using compressed-air and a steel brush.

b) The holes are filled with the chemical adhesive, and the fasteners are then inserted up to a specific depth.

c) A rough concrete surface is assured using a rotary hammer with a needle adapter. Compressed-air is used to clean the final surface.

d) A transparent acrylic strip is used to mark the fasteners position and, then, the holes in the laminates are executed. The laminates are cleaned with acetone.

e) Epoxy adhesive is applied on the treated area in the concrete surface and on the laminate surface that will be in contact.

f) The laminate is placed on the concrete surface and pressed against it to create a uniform thickness of 1-2 $\mathrm{mm}$.

g) The adhesive in excess is removed and the fasteners are cleaned from any dirt attached.

h) h. The pre-defined pre-stress level is applied after the curing time of the epoxy adhesive.

"Fig.4" shows a strengthened cross section with MF-EBR method.

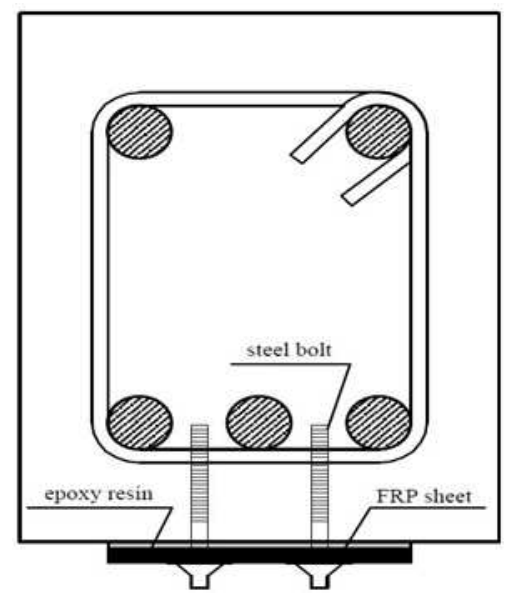

Figure 4. A strengthened cross section with MF-EBR method

\subsection{Advantages}

a) No need for special labor skills

b) No need for surface preparation

c) Increase in the load carrying capacity up to $87 \%$ compared to that of reference specimen

d) Increase in the ductility index compared to that of EBR methid

e) Immediate use of strengthened structures

\subsection{Disadvantages}

a) The larger initial cracks due to fasteners in high strength concretes

b) Low efficient stress transferability between the FRP and the concrete due to the separated connection points

c) Vulnerability of FRP materials against the environmental 
conditions

d) Changes caused in the appearance of the structure

\section{The HOLING Method}

\subsection{General Features}

Eftekhar and Yaqubi [12] suggested a new technique as an alternative method for conventional surface preparations. This technique was named HOLING method. In this method first holes of specified depth, diameter and distance from each other are drilled onto the tension side of the concrete member. These holes are then cleaned by air jet and filled with the epoxy resin. FRP sheets are later installed on the concrete surface saturated with the epoxy resin and the resin in excess is removed. This technique provides larger contact area between the concrete and epoxy resin as well as cylindrical anchors at the interface of FRP sheet and concrete. These cylindrical anchors transfer the interfacial stresses between FRP and concrete surface to the strong underlying concrete layers. This technique can postpone the debonding phenomenon and increase the ultimate load and strain [12].

"Fig.5", shows the holes drilled on the tension side of a beam in HOLING method.

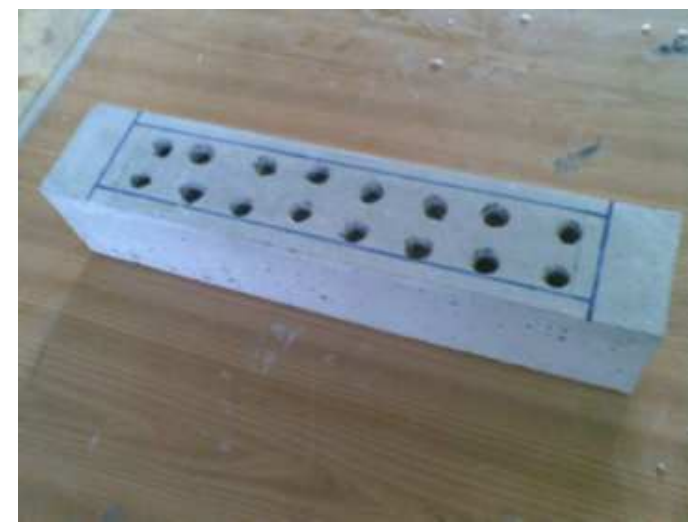

Figure 5. Holes drilled on the tension face in HOLING method

\subsection{Advantages}

a) Increase in the flexural capacity up to $30 \%$ more than EBR method

b) Increase in the ductility index and energy absorption compared to the EBR method

c) No need for special labor skills

d) Immediate use of strengthened structures

\subsection{Disadvantages}

a) Premature debonding of FRP sheet from concrete surface

b) Time-consuming drilling process

c) Costly performing process

d) Vulnerability of FRP materials against the environmental conditions

e) Environmental pollution caused by the grooving process

f) Changes caused in the appearance of the structure

\section{The NSM method}

\subsection{General Features}

As mentioned in the section 2, the EBR technique is the most common method to strengthen reinforced concrete structures. In this method, due to premature debondng of FRP sheet, the tensile capacity of FRP materials is not completely used. To overcome this defect, modifications were carried on this technique that led to invention of more efficient methods such as EBROG, EBRIG, MF-EBR and HOLING techniques. Although these method postpone or even in some cases completely eliminate the debonding phenomenon but all of them have an important problem. The problem is that the strengthening materials in these techniques are laid on the external surface of the concrete member and are vulnerable against sever environmental conditions. To overcome the mentioned weaknesses, several efforts have been done and one of the most successful methods is the near surface mounted (NSM) method [13].

The NSM method consists of the following steps:

a) Cutting grooves of specified dimensions at designed locations using a diamond blade cutter.

b) Cleaning the grooves using compressed air to remove debris and dust to ensure proper bonding between the epoxy adhesive and concrete.

c) Placing the epoxy paste into the grooves to fill $2 / 3$ of the groove depth.

d) Inserting the strengthening materials (rods or strips) in the groove and lightly pressing it to displace the adhesive, ensuring that the space between the rod or strips and the sides of the groove is completely filled with epoxy without any voids.

e) Placing additional epoxy paste to ensure that the grooves are completely filled and then levelling the surface by removing excessive adhesive.

f) Curing the members at room temperature for at least 5 days [14].

"Figs.6, 7" show two strengthened cross sections with NSM method using FRP rods and FRP strips.

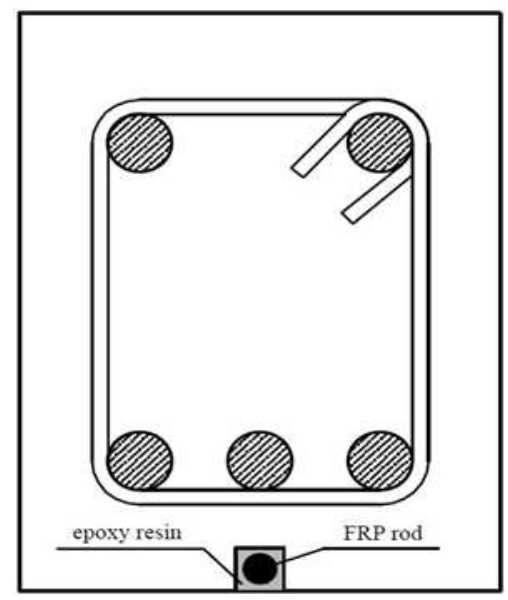

Figure 6. Application of FRP rod in NSM method 


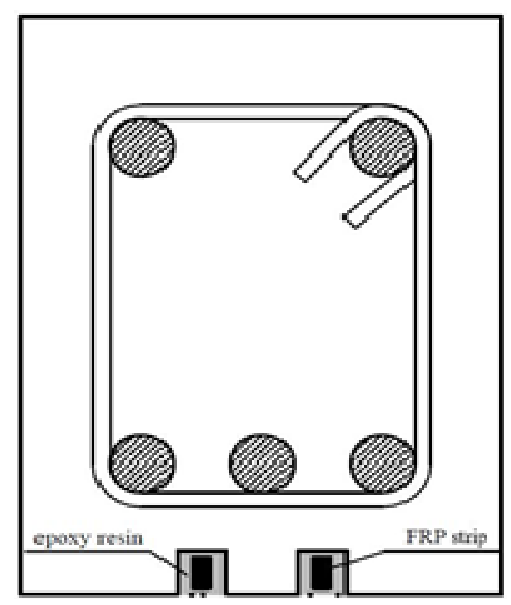

Figure 7. Application of FRP strips in NSM method

\subsection{Advantages}

a) Reduction in the strengthening operation because of no need for the surface preparation

b) Postponing or even eliminating the debonding phenomenon

c) Possibility of using this technique in the negative moment region of flexural frames because of preserving FRP materials against abrasion

d) Allowing easier pre-stressing strengthening materials

e) Preserving strengthening materials against sever environmental conditions such as: mechanical impacts, abrasion, fire, freeze/thaw cycles, vandalism and UV radiations

f) Possibility of using various types of FRP materials such as: FRP rods, FRP strips and hand-made FRP rods

\subsection{Disadvantages}

a) Costly and time-consuming installation process

b) Environmental pollution caused by the grooving process

\section{Application of MMFRP rods in the NSM method}

\subsection{General features}

The Manually Made Fiber Reinforced Polymers (MMFRP) rods are made from FRP sheets wrapping around a wooden rod. They provide a larger perimeter with respect to conventional FRP bars for the same amount of fiber as the core of the MMFRP rod consists of a low strength wooden rod, providing larger bond circumference and thus potentially higher bond strength. The circular shape of MMFRP rod is not only convenient for production but also suitable for NSM shear strengthening as noted by Rizzo and De Lorenzis [15]. The key advantage of the MMFRP rod, however, is that it allows the incorporation of a novel anchor system that can be used to improve the performance of NSM MMFRP reinforcement for shear strengthening of RC beams with low FRP percentage. This cannot be easily achieved using conventional pultruded
FRP bars or strips [16].

"Figs.8, 9, 10" show a strengthened cross section with NSM-MMFRP rod, the manufacturing procedure of MMFRP rod and it's anchorage system respectively.

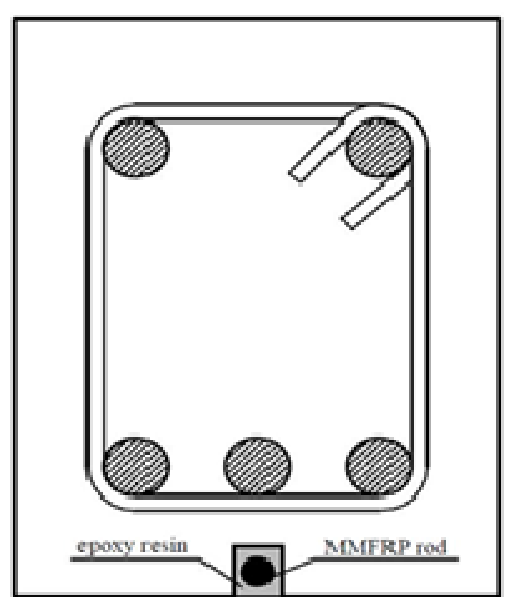

Figure 8. Application of MM FRP strips in NSM method

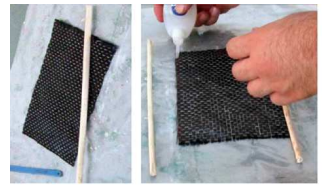

(a) (b)

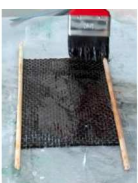

(c)

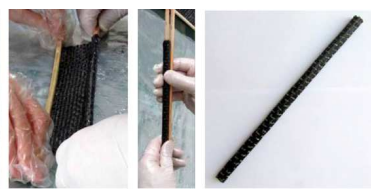

(d) (e)
Figure 9. Manufacturing procedure of MMFRP rod [16]

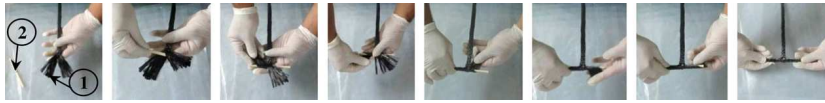

Figure 10. Fabrication procedure of MMFRP end anchorage [16]

\subsection{Advantages}

a) Possibility of mounting anchorage system at the ends of the rod in order to improve the performance of these rods

b) Possibility of producing rods of various diameters

c) Possibility of using the flexural capacity of core in the cases of steel cores

d) lower cost compared to conventional FRP rods

\subsection{Disadvantages}

a) Time-consuming manufacturing process

b) Lower quality than that produced in the factories

c) Possibility of core corrosion or core decay.

d) Environmental pollution caused by the grooving process

\section{Discussion and Conclusion}

The EBR method despite the easy and quick installation process, is faced with serious challenges such as premature debonding of the FRP sheet from the concrete surface and vulnerability against the sever environmental conditions.

The conventional surface preparation processes such as surface roughening by sandblast, air jet and water jet can slightly postpone the debonding phenomenon but cannot 
eliminate it.

EBROG and EBRIG techniques can significantly increase the load carrying capacity of concrete members. Although these techniques, postpone or even in some cases eliminate the debonding phenomenon but are faced with problems such as vulnerability against sever environmental conditions and environmental pollution caused during the grooving process.

The MF-EBR method which combines the mechanical fastening of MF-FRP method with the external adhesion in EBR method, increases the load carrying capacity and ductility index of strengthened member compared to the EBR method. However, in this method the stress is transferred with low efficiency because of separated connection points. Also in this method the FRP materials are vulnerable against sever environmental conditions.

The HOLING method can increase the load carrying capacity of strengthened members but is faced with problems such as: premature debonding, environmental pollution causedduring the holing process and time-consuming installation process.

The NSM family methods postpone or even eliminate the debonding phenomenon. Also in these methods the strengthening materials are interred in the concrete cover and are confined with the concrete sides of the grooves. Therefore these materials are preserved against sever environmental conditions. Various types of FRP materials such as FRP rods, FRP strips and MMFRP rods can be used in the NSM method. Environmental pollution caused during the grooving process is an important problem in this method.

The key advantage of the MMFRP rod, is that it allows the incorporation of an anchor system that can be used to improve the performance of NSM method.

\section{References}

[1] A. Carolin, "Carbon Fiber Reinforced Polymers for Strengthening of Structural Elements," Doctoral Thesis, Lulea University of Technology, Sweden, p. 247, 2003.

[2] H. Nordin, " Fiber Reinforced Polymers in Civil Engineering," $\mathrm{PhD}$. Thesis, Lulea University of Technology, Sweden, p. 57, 2003.

[3] S. M. Soliman, "Flexural Behaviour of Reinforced Concrete Beams Strengthened with Near Surface Mounted FRP Bars," Ph.D Thesis, University of Sherbrooke, Sherbrooke, Quebec, Canada, p. 12, 2008.

[4] M. Arduini, A. Nanni, "Behavior of Precracked RC Beams Strengthened with Carbon FRP Sheets," Journal of Composites for Construction, Vol. 1, No. 2, pp. 63-70, 1997.
[5] D. M. Nguyen, T. K. Chan,H. K. Cheong, "Brittle Failure and Bond Development Length of CFRP-Concrete Beams, " ASCE, Journal of Composites for Construction, Vol. 1, No. 5, pp. 7-12, 2001.

[6] P. Mukhopadhyaya, N. Swamy, "Interface Shear Stress: A New Design Criterion for Plate Debonding," ASCE, Journal of Composites for Construction, Vol. 1, No. 5, pp. 35-43, 2001.

[7] N. F. Grace, G. A. Sayed, A. K. Soliman, K. R. Saleh, "Strengthening of Reinforced Concrete Beams Using Fiber Reinforced Polymer (FRP) Laminates," Journal of Structures, ACI, Vol. 5, No. 96, pp. 865-74, 1999.

[8] D. Mostofinejad, E. Mahmoudabadi, "Grooving as an Alternative Method of Surface Preparation to Postpone Debonding of FRP Laminates in Concrete Beams," Journal of Composites for Construction, ASCE, V. 6, No. 14, pp. 804-11, 2010.

[9] D. Mostofinejad, S. M. Shameli, "Externally Bonded Reinforcement In Grooves (EBRIG) Technique to Postpone Debonding of FRP Sheets in Strengthened Concrete Beams," Journal of Construction and Building Materials, Vol. 38, pp. 751-758, 2013.

[10] F. Micelli, A. Rizzo, D. Galati, "Anchorage of Composite Laminates in RC Flexural," Journal of Composites for Construction, Vol. 3, No. 11, pp. 117-26, 2010.

[11] J. M. Sena-Cruz, J. A. O. Barros, M. R. F. Coelho, L. F. F. T. Silva, "Efficiency of Different Techniques in Flexural Strengthening of RC Beams under Monotonic and Fatigue Loading," Construction and Building Materials, No. 29, pp. 175-182, 2012.

[12] M. R. Eftekhar, M. Yaqubi, "Holing method to postpone debonding in strengthened RC beams with FRP," $4^{\text {th }}$ annual national conference of concrete, Iran, 2012.

[13] A. Hajihashemi, D. Mostofinejad, M. Azhari, "Investigation of RC Beams Strengthened with Prestressed NSM CFRP Laminates," ASCE, Journal of Composites for Construction, Vol. 6, No. 15, pp. 887-95, 2011.

[14] L. De Lorenzis, A. Nanni, "Shear Strengthening of Reinforced Concrete Beams with Near-Surface Mounted FRP Rods," ACI Structural Journal, Vol. 98, No. 1, pp. 60-68, 2001.

[15] A. Rizzo, L. De Lorenzis, "Behavior and capacity of RC beams strengthened in shear with NSM FRP reinforcement," Journal of Composites for Construction, Vol. 5, No. 2, p. 114, 2001.

[16] M. Jalali, M. K. Sharbatdar, J. Fei-Chen, F. Jandaghi Alaee, "Shear Strengthening of RC Beams Using Innovative Manually Made NSM FRP Bars," Construction and Building Materials Journal,Vol. 36, pp. 990-1000, 2011. 